\title{
Experiencia de manejo de cáncer pancreático con Whipple laparoscópico en el Hospital General y Central de Chihuahua
}

\author{
Experience in handling pancreatic cancer with laparoscopic \\ Whipple at the General and Central Hospital of Chihuahua
}

\author{
César Aragón Quintana,* Javier Antero Guevara López,** Juan Carlos Casas García,*** \\ Adriana Castillo Frausto****
}

Palabras clave: Cáncer de páncreas, cirugía de Whipple o pancreatoduodenectomía, tratamiento del cáncer resecable de páncreas, pancreatoduodenectomía abierta versus laparoscópica.

Key words:

Pancreatic cancer, Whipple's surgery or pancreatoduodenectomy, treatment of resectable pancreatic cancer, open versus laparoscopic pancreatoduodenectomy.

\footnotetext{
* Jefe de Servicio de Cirugía General en el Hospital General "Dr. Salvador Zubirán Anchondo" y adscrito del Servicio de Cirugía General en el Hospital Central de Chihuahua. ** Adscrito del Servicio de Cirugía del Hospital Central de Chihuahua. *** Adscrito a Oncocirugía del Centro Estatal de Cancerología. **** Residente de $4^{\circ}$ año del Hospital Central de Chihuahua.
}

Departamento de Cirugía General, Hospital Central del Estado de Chihuahua y Hospital General del

Estado de Chihuahua.

Recibido: 11/09/2016 Aceptado: 31/10/2017

\section{RESUMEN}

Los cánceres de páncreas son neoplasias malignas usualmente agresivas con altas tasas de mortalidad, pueden ser sólidas o quísticas. Las neoplasias pancreáticas sólidas más comunes son el adenocarcinoma ductal pancreático (ACDP) y tumores neuroendocrinos pancreáticos (TNEP). En México el cáncer de páncreas es la quinta neoplasia más frecuente en hombres y la séptima en mujeres. La presentación clínica de cáncer de páncreas depende principalmente de la localización del tumor dentro del páncreas. La mayoría (85\%) de los tipos de cáncer pancreáticos se desarrollan dentro de la cabeza del páncreas. Alrededor de $10 \%$ se producen en el cuerpo del páncreas y $5 \%$ en la cola. El diagnóstico de cáncer de páncreas puede ser difícil de establecer en pacientes que se presentan con ictericia obstructiva, la sospecha de cáncer de páncreas es muy alta, de manera que se realizan diversos estudios de imagen como ecografía abdominal o tomografía axial computarizada (TAC) de abdomen o ambos. La cirugía de Whipple o pancreatoduodenectomía es la única terapia potencialmente curativa para el tratamiento del cáncer resecable de páncreas. La tasa de mortalidad operatoria en cirujanos experimentados es inferior a 5\% y de 20 a 30\% en cirujanos no experimentados. Las muertes postoperatorias se deben a complicaciones como fístulas pancreáticas y biliares, hemorragia e infección. Los estudios de comparación entre pancreatoduodenectomía abierta (OPD) y laparoscópica (LPD) revelaron disminución de la pérdida de sangre, mayor tiempo quirúrgico, un índice similar de complicaciones postoperatorias, disminución del dolor y estancia hospitalaria corta en pacientes sometidos a LPD. En este estudio se intervinieron ocho pacientes con edad media de 53 años (52.87) y diagnóstico de cáncer de páncreas resecable. Los estudios de imagen obtenidos fueron tomografía computarizada y colangiopancreatografía retrógrada endoscópica. En cinco pacientes se realizó pancreatoduodenectomía (tiempo operatorio promedio 280 minutos y pérdida sanguínea de $837.5 \mathrm{ml}$ ), dos de los pacientes se convirtieron a cirugía abierta y dos fallecieron. Como complicaciones del procedimiento se encontraron: fístula pancreática de alto gasto, fuga biliar y abscesos intraabdominales. La estancia hospitalaria media fue de 11 días.

\section{ABSTRACT}

Pancreatic cancers are usually aggressive malignancies with high mortality rates, which can be solid or cystic. The most common solid pancreatic neoplasms are pancreatic ductal adenocarcinoma (PDAC) and pancreatic neuroendocrine tumors (PNET). In Mexico, pancreatic cancer is the fifth most common malignancy in men and seventh in women. The clinical presentation of pancreatic cancer depends primarily on the location of the tumor within the pancreas. Most (85\%) of pancreatic cancers develop inside the head of the pancreas. About $10 \%$ occur in the pancreatic body and $5 \%$ in the tail. Diagnosis of pancreatic cancer is established may be difficult to diagnose, in those patients who present with obstructive jaundice, suspicion of pancreatic cancer is very high are performed various imaging studies such as abdominal ultrasound or CT scan of the abdomen, or both. Whipple surgery or pancreatoduodenectomy is the only potentially curative therapy for the treatment of resectable pancreatic cancer. The operative mortality rate in experienced surgeons is less than $5 \%$ and high as $20 \%$ $30 \%$. Postoperative deaths are due to complications such as pancreatic and biliary fistulas, bleeding and infection. Studies comparing open pancreatoduodenectomy (OPD) and laparoscopic pancreatoduodenectomy (LPD) found decreased blood loss, longer surgical time, similar rate of post-operative complications, decreased pain and shorter length of hospital stay in patients undergoing LPD. In this study, 8 patients with a mean age of 53 years (52.87) and a diagnosis of resectable pancreatic cancer were involved. The imaging studies obtained were computed tomography and endoscopic retrograde cholangiopancreatography. Pancreatoduodenectomy was performed in 5 patients (average operating time 280 minutes and blood loss of $837.5 \mathrm{ml}), 2$ of the patients were converted to open surgery, 2 of the patients died. Complications of the procedure included: high expenditure pancreatic fistula, biliary leakage and intra-abdominal abscesses. The average hospital stay was 11 days. 


\section{INTRODUCCIÓN}

L os tipos de cáncer de páncreas son neoplasias malignas usualmente agresivas con altas tasas de mortalidad, éstas pueden ser sólidas o quísticas. Las neoplasias pancreáticas sólidas más comunes son el adenocarcinoma ductal pancreático (ACDP) y tumores neuroendocrinos pancreáticos (TNEP). Las neoplasias quísticas premalignas y malignas más comunes incluyen la neoplasia mucinosa papilar intraductal y la neoplasia quística mucinosa. ${ }^{1-3}$

En México el cáncer de páncreas es la quinta neoplasia más frecuente en hombres y la séptima en mujeres. ${ }^{4}$

El principal factor de riesgo de cáncer de páncreas es la historia de dicho cáncer en un familiar de primer grado. 5,6

La presentación clínica de cáncer de páncreas depende principalmente de la localización del tumor dentro de éste. La mayoría (85\%) de los tipos de cáncer pancreáticos se desarrollan dentro de la cabeza del páncreas. Alrededor de $10 \%$ se producen en el cuerpo del páncreas y $5 \%$ en la cola. 7,8

El diagnóstico de cáncer de páncreas puede ser difícil de establecer, sobre todo en pacientes con síntomas no específicos. En pacientes que se presentan con ictericia obstructiva, la sospecha de cáncer de páncreas es muy alta, de manera que se realizan diversos estudios de imagen como ecografía abdominal o tomografía axial computarizada (TAC) de abdomen o ambos para descartar el problema.4,9-12

Los criterios de resecabilidad son:

1. Ausencia de metástasis a distancia.

2. Ausencia de distorsión, trombosis tumoral o invasión de la vena mesentérica superior y la vena porta en estudios de imagen.

3. Presencia de planos de grasa alrededor del tronco celiaco, la arteria hepática y la arteria mesentérica superior. ${ }^{10,13-16}$

La cirugía de Whipple o pancreatoduodenectomía es la única terapia potencialmente curativa para el tratamiento del cáncer resecable de páncreas. La cirugía de Whipple implica la resección del conducto biliar común, la vesícula biliar, el duodeno, 15 centímetros de yeyuno y el páncreas hasta la mitad del cuerpo. La tasa de mortalidad operatoria en cirujanos experimentados es inferior a 5\%; sin embargo, aumenta hasta $20-30 \%$ en cirujanos no experimentados. Las muertes postoperatorias se deben a complicaciones como fístulas pancreáticas y biliares, hemorragia e infección. ${ }^{17-19}$

Los estudios de comparación entre pancreatoduodenectomía abierta (OPD) y laparoscópica (LPD) revelaron que en pacientes sometidos a cirugía LPD la pérdida de sangre disminuye, el tiempo quirúrgico es más prolongado, el índice de complicaciones postoperatorias es similar, hay disminución del dolor y la estancia hospitalaria es más corta. ${ }^{20-23}$

El presente trabajo sometió a pacientes a cirugía laparoscópica con resultados similares a los de la literatura universal.

\section{Objetivos}

Describir la experiencia del manejo de cáncer pancreático resecable con cirugía de Whipple laparoscópica en pacientes del Hospital General y Central de Chihuahua.

\section{Objetivos específicos:}

1. Conocer las características sociodemográficas y manifestaciones clínicas de los participantes.

2. Determinar los antecedentes personales de importancia.

3. Medir el tiempo promedio operatorio, la pérdida de sangre, la cosecha de ganglios linfáticos y los márgenes libres de enfermedad.

4. Determinar el tipo y frecuencia de complicaciones, así como la tasa de mortalidad de los pacientes y la estancia hospitalaria.

\section{MATERIAL Y MÉTODOS}

Se describirá la experiencia del Hospital General y Central de Chihuahua en el manejo de pacientes con cáncer resecable de páncreas con el procedimiento Whipple laparoscópico, en el periodo comprendido del 01 enero de 2011 al 31 de julio de 2015. 
Durante este periodo se intervinieron ocho pacientes (tres mujeres y cinco hombres) con diagnóstico de cáncer de páncreas y edad media de 53 años (52.87) (Cuadro l y Figuras 1 y 2).

Los estudios de imagen obtenidos fueron tomografía computarizada y colangiopancreatografía retrógrada endoscópica. En todos los pacientes se planteó el abordaje laparoscópico (Figura 3).

\begin{tabular}{lc}
\hline \multicolumn{2}{|c}{ Cuadro I. Características de los pacientes. } \\
\hline Variable & $\overline{\mathrm{X}} \pm \mathrm{DE} / \mathrm{n}(\%)$ \\
\hline Edad (años) & $52 \pm 12$ \\
Género & \\
$\quad$ Femenino & $3(38)$ \\
$\quad$ Masculino & $5(62)$ \\
Tabaquismo & $7(88)$ \\
Sí & $1(12)$ \\
No & \\
Abuso de alcohol & $7(88)$ \\
Sí & $1(12)$ \\
No & \\
Obesidad & $4(50)$ \\
Sí & $4(50)$ \\
No &
\end{tabular}

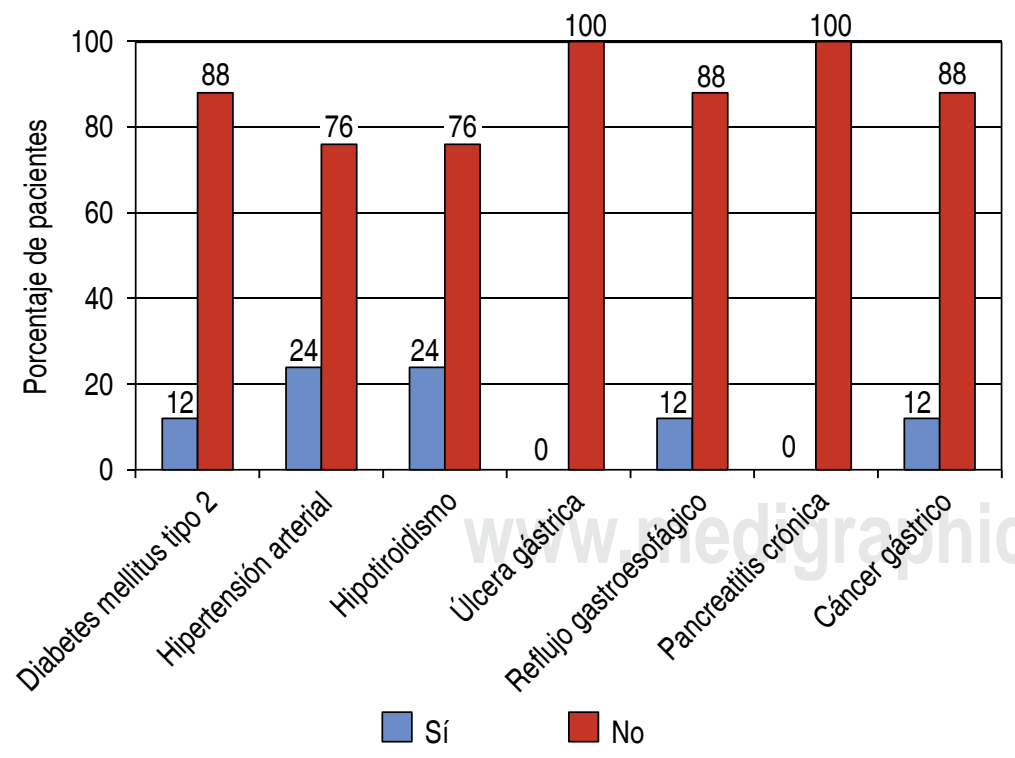

Figura 1. Antecedentes heredofamiliares y personales patológicos de los pacientes sometidos a procedimiento de Whipple laparoscópico.

\section{Criterios de inclusión}

Pacientes con cáncer resecable de páncreas, de cualquier género y edad, operados mediante procedimiento de Whipple laparoscópico en el Hospital General y Central de Chihuahua.

\section{Criterios de exclusión}

Pacientes que no contaran con datos clínicos, quirúrgicos y de seguimiento completos.

\section{RESULTADOS}

En cinco pacientes se realizó pancreatoduodenectomía (tiempo operatorio promedio 280 minutos y pérdida sanguínea de $837.5 \mathrm{ml}$ ), de los pacientes que se convirtieron a cirugía abierta (Cuadro II), dos fallecieron: uno de ellos durante el transinmediato por causas de

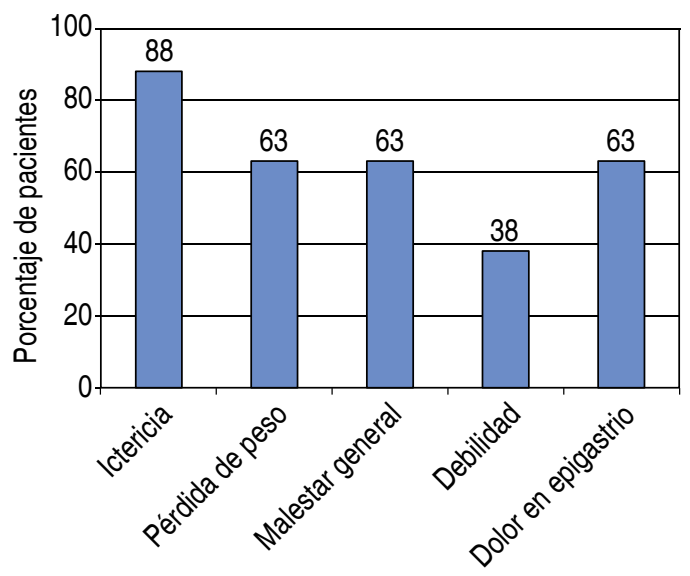

Figura 2. Manifestaciones clínicas.

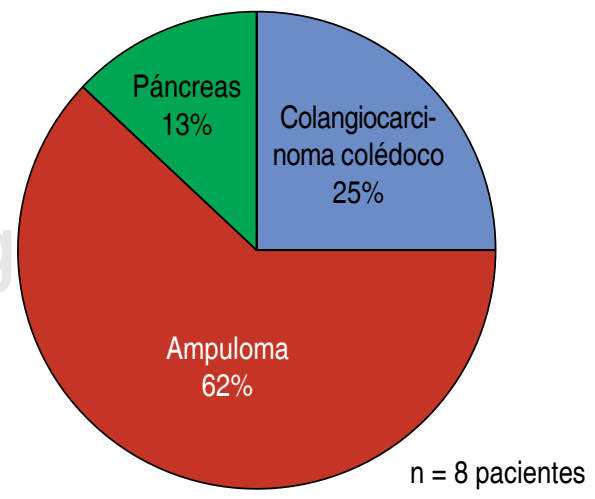

Figura 3. Diagnóstico de los pacientes. 


\begin{tabular}{lc}
\multicolumn{2}{c}{$\begin{array}{c}\text { Cuadro II. Hallazgos obtenidos } \\
\text { del procedimiento quirúrgico } \\
\text { Whipple laparoscópico. }\end{array}$} \\
\hline \multicolumn{2}{|c}{$\overline{\mathrm{X}} \pm$ DE/n (\%) } \\
\hline Variable & $308 \pm 112$ \\
\hline Tiempo quirúrgico (min.) & $1,050 \pm 979$ \\
Pérdida de sangre (mililitros) & Promedio \\
Número de ganglios linfáticos & 10 ganglios* \\
& $4(50)$ \\
Márgenes libres & $14 \pm 9$ \\
Días de estancia hospitalaria & $7(88)$ \\
Frecuencia de complicaciones & \\
\hline *n=3 pacientes. &
\end{tabular}

sangrado (lesión a vena mesentérica), el otro paciente falleció en el postoperatorio inmediato (dehiscencia de hemoclip). En el procedimiento se encontraron las siguientes complicaciones: un paciente con fístula pancreática de alto gasto, un paciente con fuga biliar y dos con abscesos intraabdominales. La estancia hospitalaria media fue de 11 días.

\section{CONCLUSIÓN}

El abordaje laparoscópico constituye una alternativa válida para el tratamiento del cáncer pancreático, mediante enucleación o resección pancreática y ofrece todas las ventajas de la cirugía mínimamente invasiva.

\section{REFERENCIAS}

1. Decker GA, Batheja MJ, Collins JM, Silva AC, Mekeel $K L$, Moss $A A$, et al. Risk factors for pancreatic adenocarcinoma and prospects for screening. Gastroenterol Hepatol (N Y). 2010; 6: 246-254.

2. Novotný I. Precancerous conditions and risk factors for pancreatic and bile duct cancer. Klin Onkol. 2013; 26: S29-33.

3. Becker AE, Hernandez YG, Frucht $H$, Lucas AL. Pancreatic ductal adenocarcinoma: risk factors, screening, and early detection. World J Gastroenterol. 2014; 20: 11182-11198.

4. Robles DG, Fastag D. Cáncer de páncreas: epidemiología y factores de riesgo. Memorias. Cáncer de páncreas. Rev Gastroenterol Mex. 2007; 72: 154-159.

5. Del Chiaro M, Segersvärd R, Lohr M, Verbeke C. Early detection and prevention of pancreatic cancer: is it really possible today? World J Gastroenterol. 2014; 20: 12118-12131.
6. Shroff RT, Wolff RA, Javle MM. Pancreatic cancer. En: Kantarjian HM, Wolff RA, Koller CA. eds. The MD Anderson Manual of Medical Oncology, Chapter 18. 2e. New York, NY: McGraw-Hill; 2011. pp. 321-339.

7. Stern SC, Cifu AS, Altkorn D. Jaundice and abnormal liver enzymes. En: Stern SC, Cifu AS, Altkorn D, eds. Symptom to diagnosis: an evidence-based guide, $3 \mathrm{e}$. New York, NY: McGraw-Hill; 2014. pp. 234-241.

8. Merkow J, Paniccia A, Edil BH. Laparoscopic pancreaticoduodenectomy: a descriptive and comparative review. Chin J Cancer Res. 2015; 27: 368-375.

9. Al-Hawary MM, Francis IR, Anderson MA. Pancreatic solid and cystic neoplasms: diagnostic evaluation and intervention. Radiol Clin North Am. 2015; 53: 1037-1048.

10. Tempero M, Arnoletti JP, Ben-Josef E, Bhargava P, Casper ES, Kim P, et al. Pancreatic adenocarcinoma. Clinical practice guidelines in oncology. J Natl Compr Canc Netw. 2007; 5: 998-1033.

11. Boyle J, Czito B, Willett C, Palta M. Adjuvant radiation therapy for pancreatic cancer: a review of the old and the new. J Gastrointest Oncol. 2015; 6: 436-444.

12. Herman JM, Swartz MJ, Hsu CC, Winter J, Pawlik TM, Sugar E, et al. Analysis of fluorouracil-based adjuvant chemotherapy and radiation after pancreaticoduodenectomy for ductal adenocarcinoma of the pancreas: results of a large, prospectively collected database at the Johns Hopkins Hospital. J Clin Oncol. 2008; 26: 3503-3510.

13. Correa Rovelo JM, Castillo V, José V. Cirugía gastrointestinal: guía práctica. México: Editorial Alfil. 2012. pp. 246-256.

14. Tempero MA, Arnoletti JP, Behrman SW, BenJosef E, Benson AB 3rd, Casper ES, et al. National Comprehensive Cancer Networks. Pancreatic adenocarcinoma, version 2.2012: featured updates to the NCCN Guidelines. J Natl Compr Canc Netw. 2012; 10: 703-713.

15. Visser BC, Ma Y, Zak Y, Poultsides GA, Norton JA, Rhoads KF. Failure to comply with NCCN guidelines for the management of pancreatic cancer compromises outcomes. HPB (Oxford). 2012; 14: 539-547.

16. Robles Díaz G, Fastag D. Cáncer de páncreas: epidemiología y factores de riesgo. Memorias. Cáncer de páncreas. Rev Gastroenterol Mex. 2007; 72: 154-159.

17. Whipple AO. The rationale of radical surgery for cancer of the pancreas and ampullary region. Ann Surg. 1941; 114: 612-615.

18. Sharma A. Chapter 28. Tumors of the Pancreas. En: Greenberger NJ, Blumberg RS, Burakoff R. eds. CURRENT Diagnosis \& Treatment: Gastroenterology, Hepatology, \& Endoscopy, 2e. New York, NY: McGrawHill; 2012. pp. 345-360.

19. Ballehaninna UK, Chamberlain RS. Biomarkers for pancreatic cancer: promising new markers and options beyond CA 19-9. Tumour Biol. 2013; 34: 3279-3292.

20. Ferlay J, Soerjomataram I, Ervik M, Dikshit R, Eser S, Mathers C, et al. GLOBOCAN 2012 v1.0, Cancer Incidence and Mortality Worldwide: IARC CancerBase No. 11 [Internet]. Lyon, France: International Agency for Research on Cancer. 2013. Available in: http:// globocan.iarc.fr. 
21. Partensky C. Epidemiology of pancreatic adenocarcinomas. Rev Prat. 2015; 65: 364-6, 369-70.

22. Doherty GM. Pancreas. En: Doherty GM, eds. CURRENT Diagnosis \& Treatment: Surgery, 14e. New York, NY: McGraw-Hill; 2015. pp. 567-575.

23. Chang DK, Johns AL, Merrett ND, Gill AJ, Colvin EK, Scarlett CJ, et al. Margin clearance and outcome in resected pancreatic cancer. J Clin Oncol. 2009; 27: 2855-2862.

\section{LECTURAS RECOMENDADAS}

- Chong I, Cunningham D. Cáncer de páncreas. En: Longo DL, Kasper DL, Jameson J, Fauci AS, Hauser SL, Loscalzo J. eds. Harrison. Principios de Medicina Interna, 18e. New York, NY: McGraw-Hill; 2012. pp. 1164

- Fisher WE, Andersen DK, Windsor JA, Saluja AK, Brunicardi F. Pancreas. En: Brunicardi F, Andersen DK, Billiar TR, Dunn DL, Hunter JG, Matthews JB, Pollock RE. eds. Schwartz's Principles of Surgery, 10e. New York, NY: McGraw-Hill; 2014. pp. 987-999.

- Rosenthal MH, Lee A, Jajoo K. Imaging and endoscopic approaches to pancreatic Cancer. Hematol Oncol Clin North Am. 2015; 29: 675-699.
- Munroe CA, Fehmi SM, Savides TJ. Endoscopic ultrasound in the diagnosis of pancreatic cancer. Expert Opin Med Diagn. 2013; 7: 25-35.

- Jackson NA, Enzinger PC. Chapter 98. Gastrointestinal Malignancies. En: Halter JB, Ouslander JG, Tinetti ME, Studenski S, High KP, Asthana S. eds. Hazzard's geriatric medicine and gerontology, 6e. New York, NY: McGraw-Hill; 2009. pp. 876-882.

- Popescu I, Dumitrascu T. Total meso-pancreas excision: key point of resection in pancreatic head adenocarcinoma. Hepatogastroenterology. 2011; 58: 202-207.

- Corsini MM, Miller RC, Haddock MG, Donohue JH, Farnell MB, Nagorney DM, et al. Adjuvant radiotherapy and chemotherapy for pancreatic carcinoma: The Mayo Clinic experience (1975-2005). J Clin Oncol. 2008; 26: 3511-3516.

Correspondencia:

Dr. César Aragón Quintana

Calle Rosales Núm. 3302

Col. Obrera Chihuahua, Chih.

Tel. 016141800800

E-mail: cesar_aragon76@hotmail.com 